\title{
Conoscere per cambiare: gli infermieri dell'Istituto Oncologico della Svizzera Italiana (IOSI) e il loro rapporto con l'Evidence Based Practice e la ricerca infermieristica
}

\author{
To know to change: the nurses of the Oncology Institute of Southern Switzerland (IOSI) \\ and their relationship with the Evidence Based Practise (EBP) and Nursing Research
}

\section{Dario Valcarenghi1 Carla Pedrazzani2 Monica Bianchi3}

\begin{abstract}
RIASSUNTO
Per favorire lo sviluppo di una cultura e di una prassi professionale basata sulle "prove di efficacia", la Direzione Infermieristica IOSI ha istituito uno specifico Ufficio, che ha deciso di definire le proprie linee d'azione in base ai risultati di una indagine interna condotta fra gli infermieri dell'Istituto, avendo come riferimento il modello dell'action research.

Agli infermieri, luglio 2010, è stato inviato un questionario semi-strutturato (self-made) per conoscere le loro opinioni ed esperienze su questi temi e per rilevare le loro proposte e la loro disponibilità a far parte di una rete di collaboratori interni. Sono tornati 63 questionari su 98 inviati $(70,8 \%)$.

Molti infermieri hanno conoscenze e/o esperienze in questi campi, ma modificano il loro comportamento professionale soprattutto su pressione del contesto esterno, più che per scelta autonoma. Considerano l'EBP sostanzialmente utile, ma di difficile applicazione soprattutto senza un loro coinvolgimento diretto. Due su tre ne hanno sentito la necessità nel corso della loro attività professionale e vi è disponibilità a sviluppare ricerche infermieristiche $(56 \%)$ e/o a svolgere un ruolo attivo di referente su questi temi nella propria sede di lavoro $(35 \%)$.

L'indagine ha evidenziato che allo IOSI esiste un substrato favorevole per l'EBP e la ricerca infermieristica (per conoscenze di base e disponibilità). I dati raccolti sono serviti a definire linee d'azione interne in uno stretto rapporto di collaborazione con le aree cliniche, secondo il modello dell'action research. E' un processo che richiede visione, interventi coordinati, costanza e tempo.

Parole Chiave: pratica basata su evidenze, ricerca infermieristica, infermiere, diffusione delle innovazioni, indagine conoscitiva, ricerca-azione
\end{abstract}

\section{ABSTRACT}

To promote the development of a culture and a professional practice based on "evidence of effectiveness", the IOSI Nursing Officer instituted a specific Unit which decided to establish its own lines of action based on the results of an internal investigation conducted among nurses employed within IOSI, with reference to the model of action research.

In July 2010, a semi-structured questionnaire self compiled was sent to all nurses to find out their opinions and experiences on EBP and nursing research, to recognize their proposals, and willingness to be part of an internal network. 63 out of 98 questionnaire were filled in.

Several nurses have knowledge and experience in these fields, but change their professional behavior especially under pressure from the external environment, rather than by autonomous choice. They consider EBP substantially useful, but difficult to implement especially without their direct involvement. Two third of the sample have felt the need of EBP during their professional activity and there is a general willingness to develop nursing research (56\%) and/or play an active role of "referent" on these issues within own Unit (35\%).

The survey showed that at IOSI there is a favorable substrate for EBP and nursing research (for basic knowledge and availability). The data collected have served to define internal lines of action in a narrow relationship with the clinical areas, according to the model of action research. It is a process that requires vision, coordinated efforts, perseverance and time.

Key words: Evidence-Based Practise, Nursing Research, Nurses, Diffusion of Innovations, Survey, Action Research

INTRODUZIONE

$\mathrm{D}$ alcuni anni, all'Ente Ospedaliero Cantonale (E.O.C.), che riunisce in un'unica gestione i sei istituti ospedalieri e riabilitativi pubblici del Canton Ticino, è stato avviato un complesso processo di quali-

1 Responsabile Ufficio Sviluppo e Ricerca Infermieristica IOSI, Bellinzona, Svizzera

2 Ufficio Sviluppo e Ricerca Infermieristica IOSI, Bellinzona, Svizzera

3 Direzione Infermieristica IOSI, Bellinzona, Svizzera ficazione e innovazione dell'area infermieristica, agendo su diversi fattori, sia di tipo organizzativo che culturale (come la riprogettazione della documentazione assistenziale e l'introduzione del modello del Primary Nursing).

Allo IOSI, che è un Dipartimento oncologico trasversale a tutte le strutture ospedaliere dell'EOC, per sostenere questi processi di cambiamento e favorire lo sviluppo di una cultura professionale e di una prassi basata sulle "prove di efficacia" e la ricerca, nell'anno 2009, la Direzione del Servizio Infermieristico ha ride- 
finito il mandato organizzativo di un pre-esistente ufficio e ne ha affidato l'incarico a un Responsabile, reclutato applicando le procedure ufficiali in uso presso l'istituto. Con l'arrivo del nuovo Responsabile (aprile 2010), sono state definite alcune linee programmatiche aventi come obiettivi principali:

- la creazione di una rete interna di referenti per la ricerca, che collaborino strettamente con l'ufficio centrale, per far circolare all'interno (in senso bidirezionale): informazioni, idee e proposte;

- la creazione di uno strumento di rapida comunicazione (uno "sharepoint" della ricerca infermieristica), per collegare fra loro tutti gli infermieri dell'istituto (sparsi in gran parte degli ospedali del Canton Ticino);

- far aumentare le conoscenze specifiche e la sensibilità degli infermieri sui temi dell'EBP, della ricerca infermieristica e del pensiero critico (con discussione periodica di "casi assistenziali" e/o articoli significativi tratti dalla letteratura);

- contribuire a stimolare e a divulgare la produzione culturale interna, fornendo supporto metodologico agli infermieri che vogliano presentare contributi ai convegni e/o pubblicare articoli su riviste professionali;

entrare e/o collaborare con reti locali, nazionali o internazionali su specifici progetti di ricerca.

Avendo come presupposto che sia possibile sviluppare una cultura basata sull'EBP e la ricerca soprattutto se i vari professionisti sanitari avranno l'opportunità di verificarne l'efficacia nella loro prassi quotidiana, si è ritenuto fondamentale partire dai problemi assistenziali realmente vissuti nell'operatività, per sottoporli a revisione critica e poter trovare insieme ai diretti interessati, anche attraverso il confronto con la letteratura esistente o un processo di ricerca, alcune possibili e concrete soluzioni operative.

Il punto di partenza fondamentale, per lo sviluppo di tutte le linee programmatiche proposte, è stato quindi quello di avere una migliore conoscenza del contesto locale. Pertanto, nell'estate 2010, è stata effettuata un'indagine interna fra gli infermieri dell'Istituto Oncologico della Svizzera Italiana, per rilevare:

le loro opinioni, conoscenze ed esperienze in tema di ricerca infermieristica ed EBP;

i loro bisogni di formazione su tali temi e sul nursing oncologico;

la loro disponibilità a svolgere un ruolo attivo, all'interno del proprio team di lavoro, per lo sviluppo delle attività di ricerca e/o la divulgazione delle "evidenze" professionali;

le loro proposte sui principali argomenti rispetto ai quali effettuare formazione, ricerca o diffusione di evidenze.

L'indagine è stata progettata nella convinzione che le conoscenze importanti che oggi servono, sono soprattutto quelle che consentono agli infermieri di prendersi cura con sempre maggiore efficacia dei malati che a loro si affidano. Le ricerche sui servizi sanitari evidenziano, però, come permanga una significativa distanza, in molti contesti di cura e assistenza, fra il mondo delle evidenze scientifiche e quello della concreta prassi professionale (Mullhall, 2002; Hanberg et al., 2006; Cartabellotta, 2010). I professionisti sanitari non sempre conoscono quali siano le migliori evidenze oggi disponibili o, se le conoscono, non sempre le applicano. Per una sorta d'inerzia professionale, si continuano ad applicare procedure inefficaci o, a volte, anche dannose per i pazienti.

Molte ricerche hanno documentato come pur a fronte di una consapevolezza ormai abbastanza diffusa sull'utilità dell'EBP, vi siano ancora solide barriere allo sviluppo e all'implementazione di un tale approccio professionale, sia fra i medici e sia tra gli infermieri, anche se con accenti diversi nelle due categorie professionali (Ya-Wen Chiu et al., 2010; Ubbink et al, 2011). Anche se vi sono studi che cominciano a porre in discussione l'eccessivo peso dato alle barriere (Brown, 2010), questo è l'orientamento tuttora prevalente.

Tra i fattori ostacolanti addotti con maggiore frequenza vi sono senźaltro: la mancanza di tempo e gli eccessivi carichi di lavoro, la mancanza di conoscenze (soprattutto metodologiche e di statistica), un atteggiamento negativo verso la ricerca, il non facile reperimento delle evidenze nel proprio contesto operativo, la mancanza di autorità per poter cambiare la pratica e la mancanza di supporto da parte della leadership professionale (Parahoo, 2000; Estabrooks et al. 2003; Hutchinson et al. 2004; Thomson et al. 2006; Lang et al. 2007; Ubbink et al., 2011; Majid et al., 2011).

Mentre fra i fattori favorenti si considerano l'investimento in formazione (richiesta in particolare dagli infermieri), una attitudine positiva verso la ricerca, la possibilità di avere dei supporti organizzativi (disponibilità di banche dati, biblioteche ospedaliere, abbonamento a riviste, ecc.), un ruolo pro-attivo della leadership infermieristica e la possibilità di un costante confronto sia fra pari che multidisciplinare (Ring, 2005; Gifford, 2007; Frasure, 2008; Halm, 2010; Ubbink et al., 2011; Einzenberg, 2011; Filippini et al., 2011). Sembra esservi un accordo abbastanza ampio sull'importanza di una formazione di alto livello per sviluppare tali competenze (McCleary et al 2003; Melnyk et al. 2004; Karlos et al. 2006; Koeln et al. 2008; Prior et al., 2010), anche se non può essere considerata come condizione sufficiente. Emblematico a tale riguardo è la studio effettuato in Svezia, nel periodo 2002-2007, sugli infermieri laureati dalle Università di quel Paese che, nonostante abbiano ricevuto una formazione speci- 
fica di alto profilo, solo in piccola parte riescono ad applicare i principi dell'EBP nella loro pratica clinica dopo circa due o tre anni di lavoro. Il processo d'implementazione dell'EBP, anche in presenza di operatori sanitari molto preparati, oltre che essere complesso, è anche molto lento ed è molto condizionato dai fattori di contesto locale e dal ruolo effettivamente assegnato alle infermiere all'interno dell'organizzazione (Boström et al., 2009).

A fronte di un sovraccarico di letteratura sanitaria, molta di utilità e qualità dubbia, per migliorare le prestazioni sanitarie sarà quindi importante riuscire a trovare vie, magari diverse, per creare solidi ponti di collegamento fra il mondo del "come si dovrebbe fare" e quello del "come si fa", favorendo un ruolo proattivo dei vari professionisti in risposta a reali problemi clinicoassistenziali e tenendo in considerazione i vincoli e le opportunità che si possono trovare in ogni contesto sanitario (McCance et al., 2007).

L'esigenza di trovare nuove forme di comunicazione e di sinergia fra teoria e prassi è spesso presente anche nei dibattiti e nelle riflessioni della comunità professionale. Una richiesta di una radicale trasformazione nella formazione infermieristica è stata, ad esempio, recentemente avanzata da alcune colleghe americane (Benner et al., 2010) che per favorire l'integrazione con la prassi clinica propongono alcuni fondamentali cambiamenti, fra i quali: il passare da una conoscenza decontestualizzata al porre l'enfasi su un insegnamento contestualizzato (pensiero e azione professionale in contesti determinati).

Secondo i noti studi di un'altra collega (Carper,1978) vi sono almeno quattro schemi fondamentali di acquisizione della conoscenza nell'assistenza infermieristica: empirico/scientifico, estetico, personale, etico. La cultura dell'EBP appartiene al primo di questi, quello empirico/scientifico, ma è importante mantenere la consapevolezza che non è l'unico modello di acquisizione della conoscenza che gli infermieri utilizzano nel loro percorso di sviluppo professionale.

Soprattutto se ci si ispira a un paradigma tendenzialmente construttivista, si comprende come solo con un "dialogo" costante con il proprio contesto clinico sia possibile avviare concreti e condivisi percorsi di cambiamento della prassi professionale, che includano anche un miglior utilizzo delle prove di efficacia.

E' il percorso avviato anche allo IOSI e che ha avuto nell'indagine interna uno dei suoi primi punti di partenza.

\section{METODOLOGIA}

Ci si è mossi seguendo, o cercando di seguire, il modello della "Action Research" (Levin, 1946) che sintetizza due momenti fondamentali: il momento teorico di studio, analisi, osservazione e conoscenza della realtà e quello pratico d'intervento, cambiamento della stessa secondo un processo integrato e dinamico. Non ci si è quindi soffermati a ricercare un approccio metodologico a prova di critica, avendo soprattutto interesse ad avviare in tempi brevi una comunicazione con il mondo locale della clinica e dell'esperienza. E' stato pertanto predisposto un questionario self-made con l'obiettivo di conoscere il contesto locale (opinioni e predisposizione verso il mondo delle "evidenze" e della "ricerca") e, nello stesso tempo, farsi conoscere (scopo e funzioni dell'Ufficio Sviluppo e Ricerca Infermieristica). Ad un obiettivo puramente conoscitivo (sviluppo di conoscenza) si è preferito un obiettivo operativo (favorire una reciproca collaborazione e creare le condizioni per lo sviluppo di una prassi basata su evidenze e ricerca).

L'utilizzo del modello dell'action research per colmare il gap esistente fra ricerca e pratica professionale trova già significativi riscontri in letteratura e, con qualche precauzione, è considerato un metodo appropriato per questo scopo (Munten et al., 2010).

A tutti gli infermieri in forza all'Istituto $\left(\mathrm{n}^{\circ} 98\right.$, con percentuali di lavoro diverse, da un minimo di 20 a un massimo di 100, con una media all'incirca del 81,5\%), nel mese di luglio 2010, è stato inviato un questionario semistrutturato, anonimo, con una lettera di presentazione a firma del Responsabile dell'Ufficio e della Responsabile della Direzione Infermieristica.

Il questionario era stato suddiviso nelle seguenti otto parti:

1 - Dati generali;

2 - Precedenti esperienze e/o formazioni su tali contenuti;

3 - Opinioni sull'utilità delle evidenze e della ricerca infermieristica;

4 - Disponibilità a formarsi su tali argomenti, in futuro;

5 - Disponibilità a svolgere un ruolo attivo,

6 - Proposte specifiche (evidenze o progetti di ricerca);

7 - Proposta di argomenti sui quali fare formazione;

8 - Valutazione sintetica finale.

La maggior parte delle domande erano chiuse (del tipo a risposta multipla) ed una parte aperte (soprattutto quelle volte a rilevare opinioni e/o proposte). In alcune domande vi era la possibilità di dare più di una sola risposta (specificato nella domanda).

Un esempio delle domande formulate (chiuse e aperte), sono di seguito riportate (Fig. 1 e 2).

Sono tornati 63 questionari dei 98 inviati, pari al $70,8 \%$. I dati sono stati inseriti in una tabella excel e analizzati in base alla loro distribuzione di frequenza e a semplici correlazioni (differenze tra infermieri esperti e non esperti, fra infermieri degli ambulatori e dei reparti). 


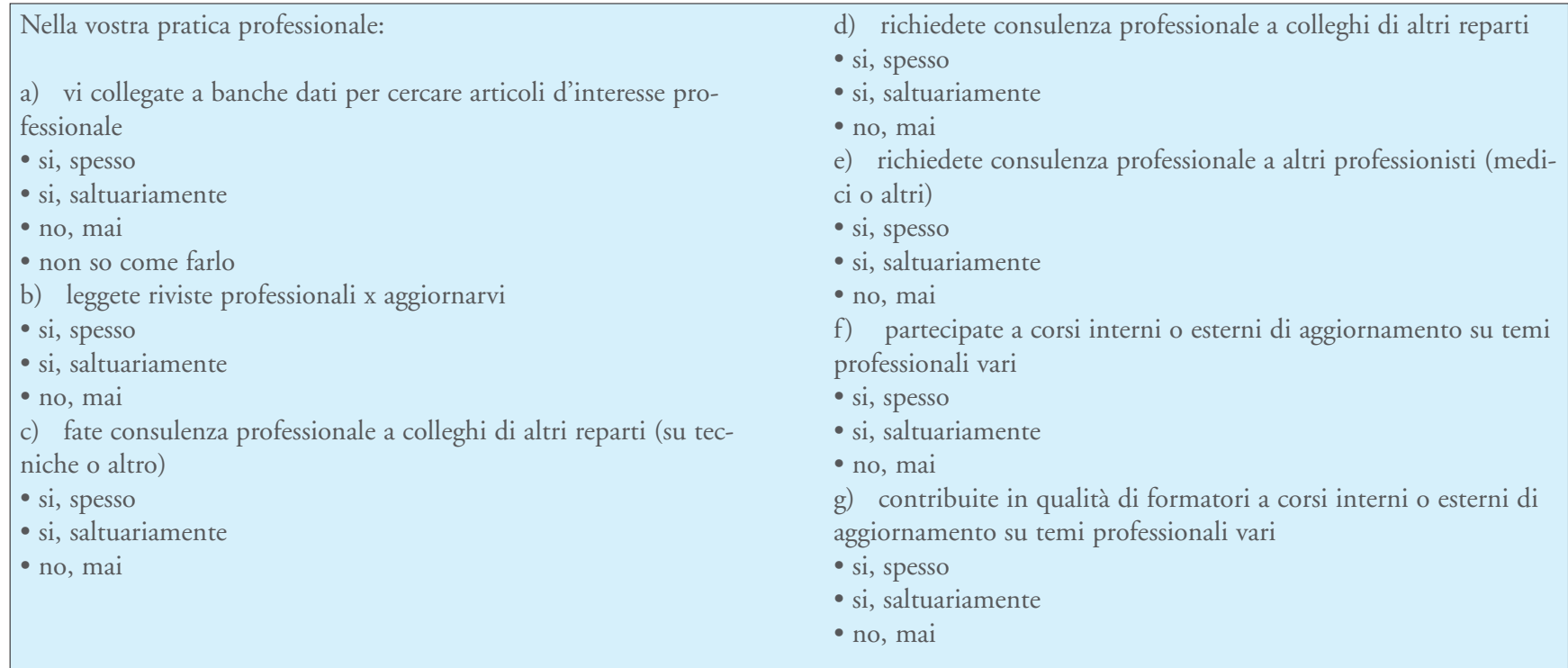

Figura 1

6.3 In base alle difficoltà che vivete nel vostro lavoro quotidiano, potreste indicare una o più situazioni problematiche che secondo voi potrebbero avere necessità di approfondire le conoscenze attraverso la ricerca di "evidenze" (articoli da ricercare sulle banche dati online) ?

6.4 In base alle difficoltà che vivete nel vostro lavoro quotidiano, potreste indicare una o più situazioni problematiche che secondo voi potrebbero avere necessità di approfondire le conoscenze attraverso un progetto di ricerca (che va oltre il reperimento di articoli già esistenti) ?

Figura 2

\section{RISULTATI}

\section{Dati generali}

La maggior parte degli infermieri che hanno risposto (grafico 1) hanno un'anzianità di servizio di altre 18 anni (35/63). Costituiscono oltre il $55 \%$ della popolazione indagata. La maggior parte lavora part-time $(57,14 \%$; 36 su 63), con percentuali oscillanti fra il 50 e l' $80 \%$. Oltre alla formazione di base, molti hanno una specializzazione in oncologia (35) o in altra area clinica (11).

La maggior parte di loro dichiara di aver avuto esperienze in ambito di ricerca o in programmi di implementazione di evidenze professionali (43 su 63, pari al $73 \%$ ). Circa la metà di loro (30 su 63) hanno partecipato all'elaborazione di protocolli e/o linee guida interne

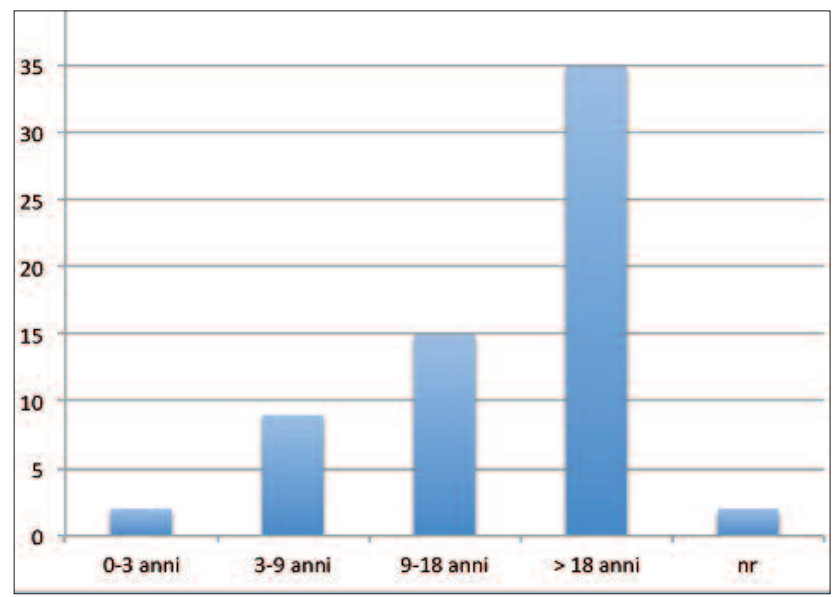

Grafico 1. Formazione e/o esperienze pregresse in ricerca (clinica o infermieristica) o nell'utilizzo di "evidenze" e un numero significativo direttamente a progetti di ricerca infermieristica (24) o clinica (18). Uno stesso numero (43) indica di aver partecipato a corsi di ricerca, probabilmente in gran parte correlati ai progetti in cui sono stati coinvolti. Gli argomenti di tali corsi sono vari e riguardano gli ambiti prevalenti di una formazione in ricerca (metodologia della ricerca, lettura critica di articoli scientifici, caratteristiche EBP, ecc.).

Dichiarano di modificare il loro comportamento professionale attraverso varie modalità, ma dalla somma delle due prime voci (spesso; a volte) si evidenzia come lo facciano soprattutto dopo: l'introduzione di protocolli (56), l'effettuazione di riunioni di reparto (54) o dopo la partecipazione a corsi di aggiornamento (54) (Grafico 2).

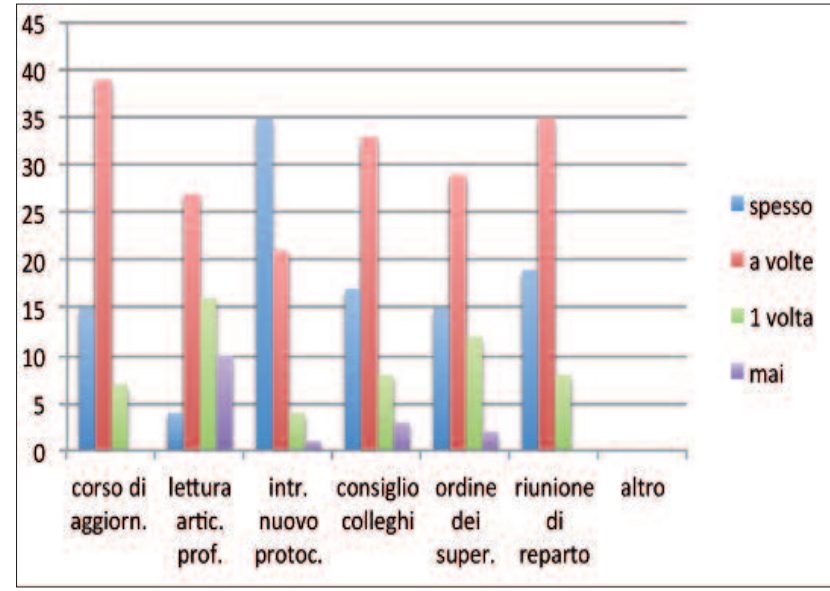

Grafico 2. Fattori che inducono a cambiare il comportamento professionale. 


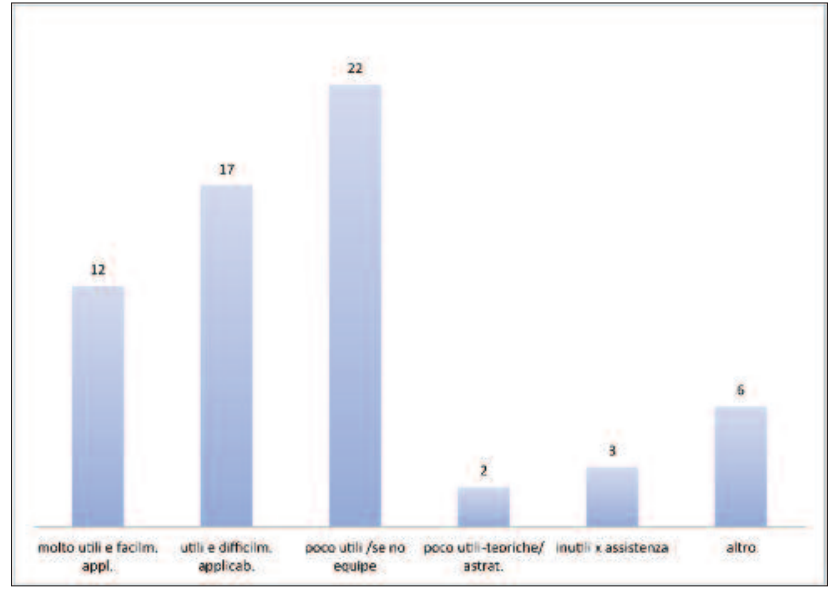

Grafico 3. Considerazioni su utilità EBN

Solo uno su cinque considera l'EBP utile e facilmente applicabile; un numero più grande $\left(n^{\circ} 17 / 63\right)$ la considera utile, ma difficilmente applicabile ed un numero ancora maggiore ( $\left.\mathrm{n}^{\circ} 22 / 63\right)$ la considera inutile senza un ruolo attivo dell'equipe che dovrebbe applicarla (Grafico 3).

Mentre la maggior parte considera decisamente utile la ricerca infermieristica come mezzo per aumentare le conoscenze professionali (35 su 63) e meno accenti critici vengono espressi in merito alla sua applicabilità (Grafico 4). A differenza dell'EBN, vista come più concreta e forse anche come non correlata con la ricerca.

Oltre i due terzi dei rispondenti (46 su 63) dichiarano di essersi trovati spesso (10) o a volte (36) in situazioni che avrebbero richiesto una revisione della letteratura o una ricerca bibliografica. Altri 15 dichiarano che questa esigenza vi è stata almeno una volta. Solo due non rispondono.

Una risposta pressoché simile viene data anche per quanto riguarda la ricerca infermieristica, dove le risposte sono state in sequenza 4 (spesso), 41 (a volte) e 16 (almeno una volta). Molto avvertita quindi è la necessità di ulteriore formazione (48 su 63, pari al 76 $\%)$, su vari argomenti necessari per poter aumentare le proprie competenze in questo ambito (Grafico 5).

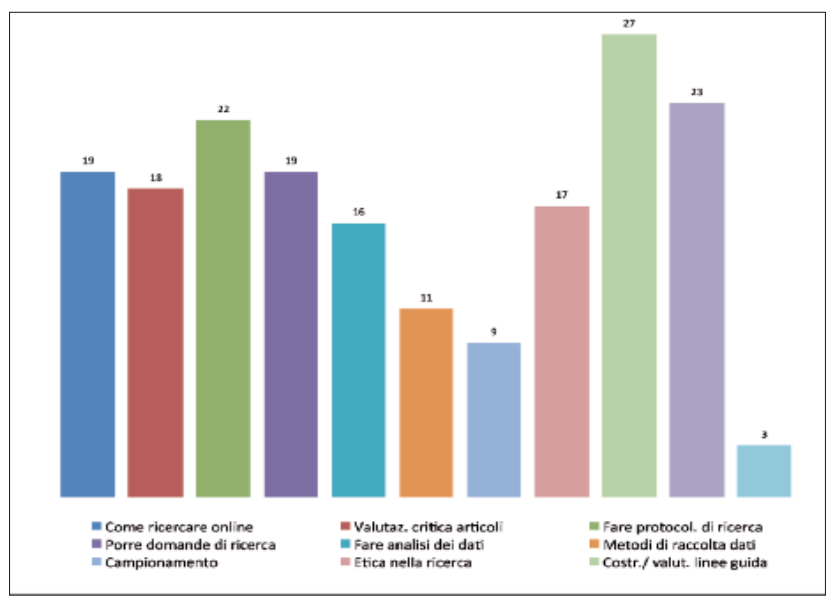

Grafico 5. Argomenti di ricerca su cui fare formazione.

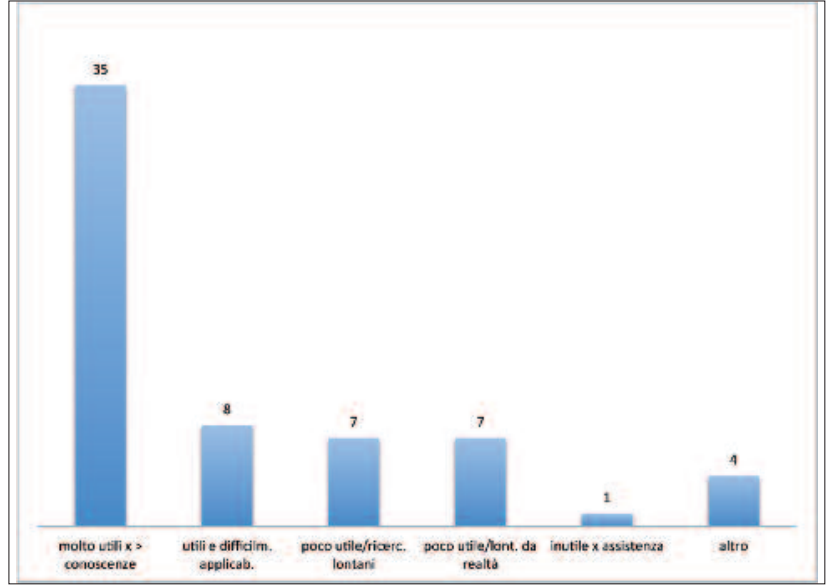

Grafico 4. Considerazioni su utilità della ricerca infermieristica

Oltre i due terzi (41 su 63, pari al $65 \%$ ) si dichiarano disponibili a partecipare al processo di diffusione di evidenze; soprattutto nel rilevare problemi su cui avviare processi di miglioramento (24), nel discutere casi assistenziali (22) o contribuire all'elaborazione di linee guida (Grafico 6).

Alta è anche la disponibilità a contribuire allo sviluppo di ricerche infermieristiche (35 su 63, pari al $56 \%$ ), soprattutto nel ruolo di coloro che possono proporre domande di ricerca.

La generica disponibilità ad essere referenti per L'EBN e la ricerca infermieristica è manifestata da 22 soggetti (pari al 22,4\% degli infermieri dello IOSI e al $35 \%$ dei rispondenti al questionario) che hanno dato il proprio nominativo per svolgere un ruolo che verrà ufficializzato tramite una lettera della Direzione Infermieristica, ottenendo così di avere in ogni Unità Operativa la presenza di almeno un referente (media $=3,6$ ), per tenere i contatti con l'ufficio centrale.

\section{Proposte in tema di evidenze e/o di progetti di ricerca}

Per quanto riguarda gli argomenti sui quali far circolare evidenze o effettuare ricerche infermieristiche, le proposte prioritarie degli infermieri IOSI (44 risposte totali, in risposta a domande chiuse) sono soprattutto

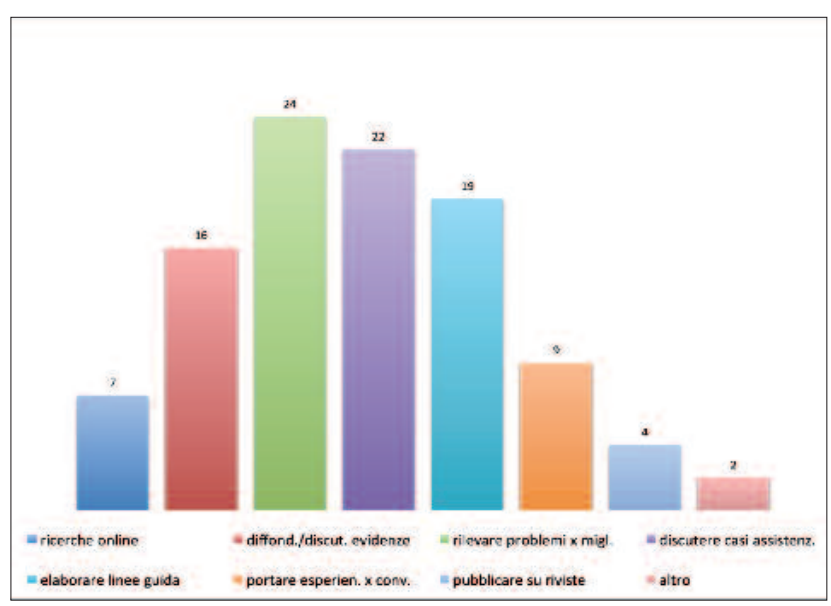

Grafico 6. Preferenze sul tipo di attività (disponibilità). 
rivolte alla gestione dei sintomi fisici (20 su 44), psicologici (26 su 44) e agli aspetti relazionali con i colleghi/superiori (21 su 44), con l'equipe (16 su 44) e con i familiari dei pazienti (16 su 44). Entrando maggiormente nel dettaglio, in risposta alle domande aperte, sono stati proposti temi quali: la gestione dei pazienti giovani (soprattutto se morenti), la relazione con il paziente anziano, l'informazione/educazione sanitaria dei pazienti, la gestione dei sintomi complessi (nausea non controllata, mucosite, danni cutanei da radioterapia, malnutrizione), la gestione dei familiari e delle loro dinamiche, la comunicazione e la relazione con gli altri membri dell'equipe multi-professionale (Grafico 7).

Un numero minore di proposte sono state raccolte per quanto riguarda dei possibili progetti di ricerca (16 risposte totali), raggruppabili in 4 aree principali: problemi tecnico-professionali (5), problemi di ruolo professionale (4), problemi organizzativo-professionali (6), problemi nella gestione dei pazienti e dei loro familiari (5). In risposta alle domande aperte, sono stati proposti argomenti quali: la gestione dei pazienti aggressivi, l'utilità delle "family conferenze" nelle cure palliative, come percepiscono i pazienti le "direttive anticipate", l'utilizzo di nuovi sistemi per l'infusione venosa centrale (PICC), la gestione della chemioterapia a domicilio, l'utilità di strumenti di assessment per l'anamnesi infermieristica, l'utilità della "consulenza infermieristica, la valorizzazione del ruolo infermieristico, la prevenzione dei rischi per i pazienti e gli operatori.

\section{Proposte relative ad altre esigenze formative (in area oncologica)}

Nell'indagine sono state anche rilevate le esigenze relative alla formazione in area oncologica, che è stata segnalata come necessaria dal $77,7 \%$ di coloro che hanno risposto. Le risposte sono state analizzate nel loro insieme e in modo separato per gli infermieri operanti in ambulatorio e per quelli operanti in reparto. le maggiori esigenze formative riguardano soprattutto

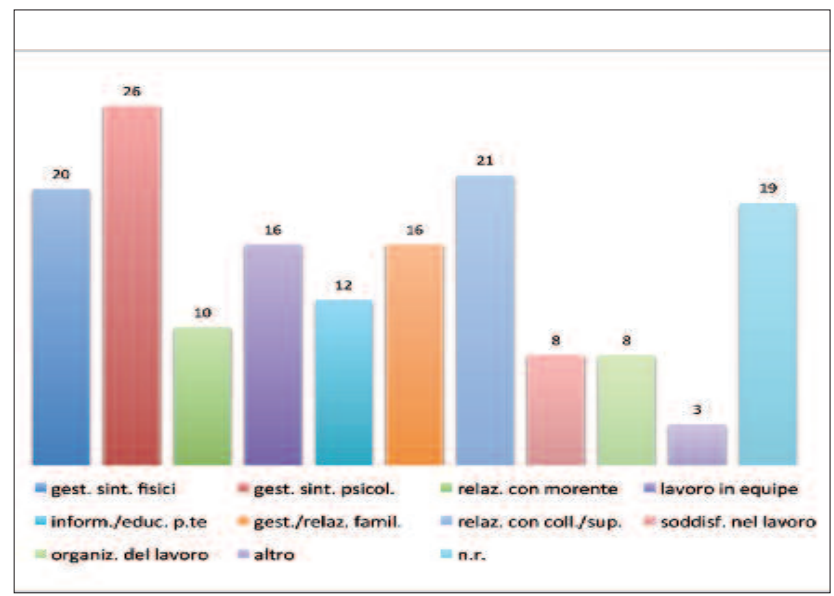

Grafico 7. Argomenti prioritari per EBN e ricerca infermieristica tre aree: i nuovi trattamenti in ambito oncologico, l'evoluzione delle conoscenze nelle patologie oncologiche e la gestione delle problematiche emotive dei pazienti (e in subordine anche dei familiari). Vi sono bisogni formativi diversi fra chi lavora in ambulatorio e chi lavora in reparto. In ambulatorio, rispetto ai reparti, prevalgono le esigenze formative sulle patologie oncologiche ed i nuovi trattamenti oltre che su ciò che riguarda la relazione con il paziente (Grafico 8).

\section{Valutazione sintetica}

A fine questionario si è chiesto di dare una valutazione sintetica rispetto all'importanza, alla complessità e alla priorità di tali temi (ricerca infermieristica ed EBP) per la pratica professionale. Il giudizio doveva essere espresso utilizzando una scala analogica numerica, con valori compresi fra zero (valore minimo) e dieci (valore massimo). La media delle risposte ricevute è stata di 5,8 per la priorità, 6,4 per l'importanza e 6,6 per la complessità.

\section{Alcune correlazioni}

Le risposte ottenute sono state anche analizzate provando a fare due correlazioni fra variabili ritenute a priori particolarmente significative.

La prima correlazione verificata, è il rapporto con l'età di servizio, ipotizzando che vi potesse essere una diversa predisposizione verso i temi dell'EBP e della ricerca infermieristica in funzione di questa variabile (e quindi anche della formazione ricevuta in anni diversi).

Di fatto fra i vari gruppi di età di servizio non sono state osservate differenze particolarmente significative, probabilmente perché molti di coloro che avevano una età di servizio superiore a 18 anni, pur avendo fatto una formazione di base in anni e anche in Paesi diversi, hanno poi fatto una formazione specialistica (25 su 35) a livello locale con modelli culturali e professionali simili. Gli infermieri con maggiori anni di servizio hanno solo evidenziato in modo particolare l'impor-

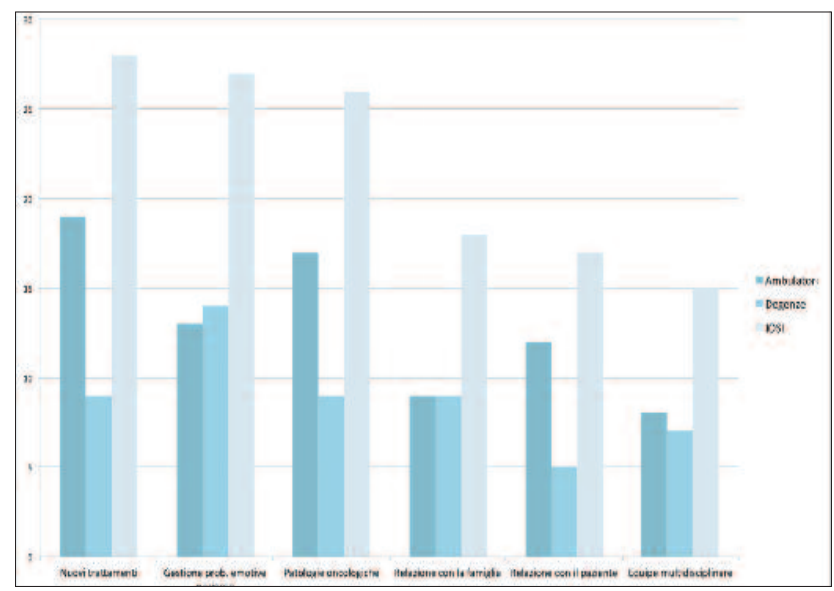

Grafico 8.Argomenti prioritari di formazione 
tanza di un loro coinvolgimento nell'elaborazione delle linee guida da utilizzare nella loro unità operativa.

La seconda correlazione verificata, come precedentemente già indicato, è stata quella relativa alla sede di lavoro (reparto o ambulatorio), ipotizzando che il particolare contesto di lavoro potesse in qualche modo influenzare il rapporto degli infermieri con tali temi.

Anche in questo caso non si sono riscontrate differenze significative fra i due gruppi di lavoro in merito alle loro opinioni o alla loro esperienza con il mondo delle "evidenze" e della ricerca. Le differenze che si sono rilevate sono state soprattutto relative alle proposte formative e ai contenuti di possibili futuri progetti di ricerca, essendo differente il focus dei problemi professionali vissuti dai due gruppi di lavoro.

\section{DISCUSSIONE}

L'indagine effettuata aveva un obiettivo soprattutto operativo: indagare il rapporto esistente fra gli infermieri dello IOSI ed il "mondo delle evidenze" al fine di meglio orientare l'attività dell'Ufficio Sviluppo e Ricerca Infermieristica che si era da poco costituito.

Da questa indagine emerge che la popolazione infermieristica dello IOSI ha prevalentemente una lunga esperienza professionale e, pur avendo avuto in maggioranza una formazione in anni in cui il tema delle evidenze non era ancora percepito nella sua importanza, ha avuto l'opportunità di affrontare tali argomenti partecipando direttamente a ricerche sul campo oppure a specifici corsi di aggiornamento. In questo possono aver influito sia le caratteristiche dell'Istituto, dove si fa anche molta ricerca clinica, e la scelta della Direzione Infermieristica di avere tendenzialmente personale "specializzato" in oncologia. Ben 46 infermieri sui 63 che hanno risposto, hanno infatti una specializzazione in ambito clinico (di cui 35 in oncologia) e nei curricula formativi di tali corsi vi sono parti importanti teorico-pratiche dedicate ad acquisire conoscenze e competenze specifiche nel mondo delle "evidenze" e della ricerca. Come evidenziato da alcuni Autori, la cultura organizzativa in cui gli infermieri lavorano ha un ruolo importante nell'influenzare l'orientamento verso la ricerca ed il suo utilizzo nella pratica clinica (Chummun et al. 2008).

Probabilmente anche per queste ragioni, vi è una diffusa sensibilità su tali temi. L'EBP è considerata generalmente utile, ma certamente non di facile applicazione. Per il suo reale utilizzo fondamentale è ritenuto essere il coinvolgimento diretto dell'equipe che dovrà applicarla. Ancora più utile rispetto all'EBP, è considerata la ricerca infermieristica come mezzo per aumentare le conoscenze professionali (35 su 63) e meno accenti critici vengono espressi in merito alla sua appli- cabilità. In questo forse vi può essere ancora un eco della concezione che la ricerca sia un problema prevalentemente accademico e quindi non immediatamente fruibile nella prassi professionale (a differenza dell'EBP, vista come più concreta anche se non facilmente applicabile). In questa difformità di risposte si può intravvedere come ancora molti colleghi non percepiscano come fortemente correlate queste due attività.

Il cambiamento del proprio comportamento professionale, che può essere determinato da molti fattori, sembra avvenire più per effetto della pressione del contesto esterno (introduzione di protocolli, riunioni di reparto, consiglio dei colleghi, ordine dei superiori) piuttosto che per autonoma decisione personale. Se ne può dedurre che, per una efficace strategia di cambiamento, siano molto importanti le decisioni assunte da coloro che hanno il potere organizzativo d'introdurre delle innovazioni all'interno dell'Istituto. Le quali, a loro volta, possano fungere da "acceleratori" di una evoluzione professionale, che può essere tanto più rapida ed efficace quanto più vi è un terreno fertile $\mathrm{e}$ culturalmente predisposto a reagire a tali cambiamenti. Anche da ciò si può desumere l'importanza della leadership professionale, come già evidenziato in alcuni studi (Halm, 2010; Everett et al., 2011). Consapevoli che lo sviluppo di un approccio professionale infermieristico basato sulle "evidenze", anche dove vi siano condizioni ottimali, è comunque da considerarsi un processo lento e complesso (Boström et al. 2009). Fondamentale sembra essere l'adozione di una strategia d'implementazione multiforme e di lungo periodo, che ha nella leadership professionale il "motore" e la principale garanzia.

Nel corso della loro esperienza di lavoro quasi tutti coloro che hanno risposto al questionario si sono trovati, con frequenza diversa, in situazioni che avrebbero richiesto un supporto attraverso la via della ricerca o dell'EBP. Molto avvertita quindi è anche la necessità di ulteriore formazione (48 su 63, pari al $76 \%$ ), sui vari argomenti che possono aiutare ad aumentare le proprie competenze in questo ambito.

Molti hanno espresso una generica disponibilità a contribuire alla diffusione delle evidenze e circa un infermiere ogni quattro si è reso disponibile a svolgere lo specifico ruolo di "referente" all'interno della propria unità operativa. Vi è in particolare la disponibilità a essere dei "rilevatori di problemi", a partecipare alla discussione di casi assistenziali e all'elaborazione di linee guida. Mentre gli argomenti sui quali si ritiene necessario produrre o far circolare "evidenze" sono soprattutto quelli relativi alla gestione dei sintomi (fisici e psicologici) dei pazienti e ad alcuni aspetti relazionali (con colleghi e superiori, con l'equipe e con i familiari dei pazienti).

Molto alto è anche il fabbisogno formativo su vari 
argomenti dell'area oncologica, che è espresso da circa 4 infermieri su 5 , per un settore della medicina e dell'assistenza che è in continua evoluzione e che oltre ad aspetti tecnico-scientifici, sollecita tematiche emotive e relazionali delicate e complesse.

In sintesi, emerge un quadro generale caratterizzato dall'esistenza di un substrato favorevole allo sviluppo di una prassi professionale basata sulle evidenze, che potrebbe anche supportare dei possibili percorsi di ricerca infermieristica a livello locale. Tale substrato, è il frutto di una serie di pregresse e lungimiranti decisioni assunte dalle Direzioni generali e professionali dell'Istituto che hanno sempre favorito una cultura delle "prove di efficacia" (anche se poi si è sviluppata soprattutto in ambito clinico-farmacologico) e l'investimento in formazione, collaborando con l'Università locale per la formazione di infermieri specializzati in area oncologica (come in altre aree cliniche).

Quello che è mancato finora, in questo processo, è l'avere risorse dedicate a promuovere l'impiego di tale cultura all'interno della prassi operativa quotidiana, trovando con costanza e coerenza le modalità più opportune per sviluppare e consolidare tale approccio professionale. Problema in gran parte risolto allo IOSI con la costituzione di apposito ufficio, inserito nella struttura organizzativa della Direzione Infermieristica e che opera in sinergia ed integrazione con esso.

In una situazione favorevole, con risorse dedicate e con il forte sostegno della leadership professionale, il problema è allora soprattutto quello di trovare le linee d'azioni più efficaci per conseguire tale scopo. Anche in questo campo è importante avere una "visione", essere determinati a seguirla e saper sfruttare le opportunità che ogni contesto culturale ed organizzativo, in una misura più o meno grande, può offrire. Pur nella consapevolezza che l'acquisizione della conoscenza e lo sviluppo di competenze nell'assistenza infermieristica è fenomeno troppo complesso per ridursi alla semplice applicazione di un unico modello (Carper, 1978) e che tra infermieri novizi ed infermieri esperti vi sono sostanziali differenze nell'approccio ai problemi del paziente (Benner et al. 1992).

Non vi può pertanto essere un'unica e monolitica proposta organizzativa per contesti diversi e dinamici, ma vi può essere una comune tensione a ricercare un "dialogo" profondo fra teoria e prassi, realizzandolo in forme e modalità congruenti con le specificità di ogni situazione locale.

Per concludere, allo IOSI, la principale funzione dell'Ufficio Sviluppo e Ricerca infermieristica, operando in sinergia con la Direzione Infermieristica sarà soprattutto quella di fungere da ponte di collegamento fra il "mondo delle evidenze" e lo "specifico contesto clinico". Facilitando la comunicazione fra queste due dimensioni ancora troppo distanti, nell'interesse di coloro che dovranno essere assistiti. Non è un risultato che possiamo considerare certo, ma vi è l'impegno e vi sono i presupposti per farlo diventare molto probabile.

\section{LIMITI}

Per raccogliere i dati è stato utilizzato un questionario self-made che, se ha avuto il vantaggio di essere costruito in base agli scopi dell'indagine svolta, ha però il grosso limite di non consentire un confronto con altre indagini simili svolte in contesti differenti.

Lo scopo principale dell'indagine è stato quello di raccogliere alcuni dati su aspetti diversi (conoscenze ed esperienze, bisogni di formazione, disponibilità a svolgere un ruolo attivo, proposte per ricerche o per l'implementazione di evidenze), in modo relativamente semplice, per poter definire alcune linee d'azione dell'Ufficio Sviluppo e Ricerca Infermieristica che fossero quanto più possibile in sintonia con il contesto locale. Le informazioni raccolte sono poi servite a orientare un multiforme processo d'implementazione della cultura dell'EBP all'interno della prassi professionale locale, che ha già prodotto alcuni risultati tangibili (premi, ricerche, pubblicazioni) anche se non sono stati ancora raccolti dei dati sistematici che possano dimostrare l'efficacia di tutto quanto fatto finora (un limite del processo).

Il principale limite delle ricerche e delle soluzioni operative che nascono dai contesti locali è la loro specificità, ma si possono sempre trovare aspetti di carattere generale utili per una comune riflessione e che possano poi essere parzialmente "trasferiti" in altri contesti locali, con gli opportuni adattamenti.

Nella nostra esperienza ciò che riteniamo possa essere occasione di un comune confronto sono soprattutto la strategia e i riferimenti che sono stati utilizzati per promuovere all'interno del nostro istituto lo sviluppo di una cultura basata sulle prove di efficacia e sulla ricerca infermieristica, di cui l'indagine che presentiamo, pur con i suoi limiti metodologici, è stata solo un primo, ma importante passo.

"Conoscere quindi per poter cambiare"... e la relazione dialettica fra i due momenti è per noi da considerarsi come fondamentale.

\section{BIBLIOGRAFIA}

Benner, P., Tanner, C., \& Chesla, C. (1992). From beginner to expert: Gaining a differentiated clinical world in critical care nursing. ANS Advances in Nursing Science, 14 (3), 13-28.

Benner, P., Sutphen, M., Leonard, V., Day, L., \& Shulman, L.S.(2009). Educating Nurses: A Call for Radical Transformation. San Francisco, Carnegie Foundation \& 
Jossey-Bass.

Boström, A.M., Ehrenberg, A., Gustavsson, P., \& Wallin, L. (2009). Registered nurses' application of evidence-based practise: a national survey. Journal of Evaluation in Clinical Practise, 15, 1159-1163.

Brown, C.E., Ecoff, L., Kim, S.C., Wickline, M.A., Rose, B., Klimpel, K., \& Glaser, D. (2010). Multi-institutional study of barriers to research utilization and evidencebased practice among hospital nurses. Journal of Clinical Nursing, 19, 1944-1951.

Carper, B.A.(1978). Fundamental Patterns of Knowing in Nursing. ANS Advances in Nursing Science, 1 (1), 13-23.

Cartabellotta N. (2010). Modificare la pratica professionale? Yes ...we can (I e II). GIMBE News, $3(5,6)$.

Chummun, H., \& Tiran, D. (2008). Increasing research evidence in practice: A possible role for the consultant nurse. Journal of Nursing Management, 16(3), 327-333.

Eisenberg, M.M. (2011). Implementation of evidence-based nursing practise: nurses' personal and professional factors? Journal of Advanced Nursing, 67 (1), 33-42.

Estabrooks, C., Floyd, J., Scott-Findlay, S., O’Leary, K., \& Gushta, M. (2003). Individual determinants of research utilization: a systematic review. Journal of Avanced Nursing, 43, 506-520.

Everett, L.Q., \& Sitterding, M.C. (2011). Transformational leadership required to design an sustain evidence-based practise: a system exemplar. Western Journal Of Nursing Research, 33 (3), 398-426.

Filippini, A., Sessa, A., Di Giuseppe, G., \& Angelillo, I.F. (2011). Evidence-based practise among nurses in Italy. Evaluation \& The Health Professions, 34 (3), 371-382.

Frasure, J. (2008). Analysis of instruments measuring nurses' attitudes towards research utilization: A sistematic review. Journal of Avanced Nursing, 61(1), 5-18.

Gifford, W., Davies, B., Edwards, N. et al. (2007). Managerial leadership for nurses' use of research evidence: an integrative review of the literature. Worldviews Evidence Based Nursing, 4(3), 136-145.

Halm, M.A. (2010). "Inside Looking In" or "Inside Looking Out"? How Leaders Shape Cultures Equipped for Evidence-Based Practice. AJCC American Journal of Critical Care, 19(4), 375-378.

Hanberg, A., \& Brown, S.C. (2006). Bridging the theorypractice gap with evidence-based practice. Journal of Continuing Education in Nursing, 37(6), 248-249.

Hutchinson, A.M., \& Johnston, L. (2004). Bridging the divide: a survey of nurse's opinions regarding barriers to and facilitators of research utilization in the practice setting. Journal of Clinical Nursing, 13, 304-315.

Karlos, B., \& Peters, K. (2006). A magnet community hospital: Fewer barriers to nursing research utilization. Journal of Nursing Amministration, 36(7/8), 377-382.

Koehn, M., \& Lehman, K. (2008). Nurses' perceptions of evidence-based nursing practice. Journal of Avanced Nursing, 62(2), 209-215.
Lang, E., Wyer, P.C., \& Haynes, R.B. (2007). Knowledge translation: closing the evidence to practice gap. Annals of Emergency Medicine, 49(3), 355-363.

Levin, K. (1946). Action Research and minority problems. Journal of Social Issues, 2, 34-46.

Majid, S., Schubert, F., Brendan, L., Xue, Z., Yin-Leng, T., Yun-Ke, C., et al. (2011). Adopting evidence-based practise in clinical decision making: nurses' perceptions, knowledge, and barriers. Journal Of The Medical Library Association: JMLA, 99 (3), 229-236.

McCance, T.V., Fitzsimons, D., Kenney, S., Hasson, F., \& McKenna, H.P. (2007). Capacity building in nursing and midwifery research and development: An old priority with a new perspective. Journal of Avanced Nursing, 59(1), 57-67.

McCleary, L., \& Brown, G. (2003). Association between nurses' education about research and their research use. Nurse Education Today, 23(8), 556-565.

Melnyk, B., Fineout-Overholt, E., Feinstein, N.F., Li, H., Small, L., Wilcox, L., et al. (2004). Nurses' perceived knowledge, beliefs, skills, and needs regarding evidencebased practice: implications for accelerating the paradigm shift. Worldwideviews on Evidence-Based Nursing, 1(3), 185-193.

Mullhall, A. (2002). Nursing research and nursing practice: An exploration of two different cultures. Intensive \& Critical Care Nursing, 18(1), 48-55.

Munten, G., Van den Bogaard, J., Cox, K., Garretsen, H., \& Bongers, I. (2010). Implementation of Evidence-Based Practice in Nursing Using Action Research: A Review. Worlviews on Evidence-Based nursing, 7(3), 135-157.

Parahoo, K. (2000). Barriers to and facilitators of research utilization among nurses in Northern Ireland. Journal of Avanced Nursing, 31, 89-98.

Prior, P, Wilkinson, J., \& Neville, S. (2010). Practice nurse use of evidence in clinical practice: A descriptive survey. Nursing Praxis in New Zeland, 26(2), 14-25.

Ring, N., Malcolm, C., Coull, A., Murphy-Black, T., \& Watterson, A. (2005). Nursing best practice statements: an exploration of their implementation in clinical practice. Journal of Clinical Nursing, 14, 1048-1058.

Thompson, D., Chau, J., \& Lopez, V. (2006). Barriers to and facilitators of research utilization: a survey of Hong Kong registered nurses. International Journal of Evidence-Based Practice, 4, 77-82.

Ubbink, D.T., Vermeulen, H., Knops, A.M., Legemate, D.A., Oude Rengerink, K., Heineman, M.J., et al. (2011). Implementation of evidence-based practise: outside the box, throughout the hospital. The Netherlands Journal of Medicine, 69 (2), 87-94.

Ya-Wen, C., Yi-Hao, W., Heng-Lien, L., Chih-Cheng, H., Ya-Hui, S., \& Ken, N.K. (2010). Comparison of evidence-based practise between physicians and nurses: a national survey of regional hospital in Taiwan. Journal of Continuing Educational in The Health Professions, 30 (2), 132-138. 